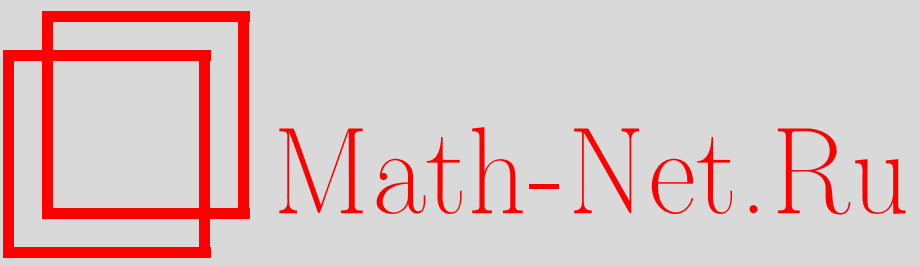

С. Х. Арансон, Е. В. Жужома, Об асимптотических направлениях полутраекторий аналитических потоков на поверхностях, УМH, 2002, том 57, выпуск 6, 169 170

DOI: https://doi.org/10.4213/rm576

Использование Общероссийского математического портала Math-Net.Ru подразумевает, что вы прочитали и согласны с пользовательским соглашением http://www . mathnet.ru/rus/agreement

Параметры загрузки:

IP : 54.197 .130 .99

26 апреля 2023 г., $14: 29: 15$ 


\title{
ОБ АСИМПТОТИЧЕСКИХ НАПРАВЛЕНИЯХ ПОЛУТРАЕКТОРИЙ АНАЛИТИЧЕСКИХ ПОТОКОВ НА ПОВЕРХНОСТЯХ
}

\author{
С. Х. АрансОН, Е. В. ЖКужома
}

Исследование нелокального асимптотического поведения полутраекторий потоков на поверхностях неположительной эйлеровой характеристики является одной из основных задач сравнительно недавно возникшей теории Аносова-Вейля (см. основные работы [1]-[5], а также обзоры $[6],[7])$. В настоящей работе описьваются нелокальные асимптотические направления поднятий на универсальню накрывающую полутраекторий аналитических потоков на замкнутой гиперболической ориентируемой поверхности.

Пусть $\Delta-$ плоскость Лобачевского в виде модели Пуанкаре, т.е. $\Delta$ - единичньй круг на комплексной $z$-плоскости, наделенный метрикой постоянной отрицательной кривизны, которая задается квадратичной формой $d s=2|d z| /\left(1-|z|^{2}\right)$. Окружность $S_{\infty}=\partial \Delta=(|z|=1)$ называется абсолютом. Геодезическими плоскости $\Delta$ являются дуги евклидовых окружностей, перпендикулярные абсолюту. Известно, что для любой ориентируемой замкнутой поверхности $M$ рода $g \geqslant 2$ найдется фуксова группа $\Gamma$ сохраняющих ориентацию изометрий плоскости $\Delta$ такая, что $\Delta / \Gamma \cong M$. Обозначим через $\pi: \Delta \rightarrow \Delta / \Gamma \cong M$ естественную проекцию, которая является универсалшным накрывающим отображением. С метрикой, индуцируемой отображением $\pi, M$ является ориентируемой гиперболической поверхностью.

Пусть $l=\{m(t) \in M: t \geqslant 0\}-$ полубесконечная непрерьвная кривая без самопересечений на $M$ и $\bar{l}=\{\bar{m}(t) \in \Delta: t \geqslant 0\}$ - ее поднятие на $\Delta$. Предположим, что $\bar{l}$ стремится в евклидовой метрике на замкнутом диске $\Delta \cup S_{\infty}$ при $t \rightarrow+\infty$ ровно к одной точке $\sigma$ абсолюта $S_{\infty}$. Будем говорить в этом случае, что кривая $\bar{l}$ имеет асимптотическое направление, определяемое точкой $\sigma$ (допуская некоторую вольнсть, будем говорить также, что $l$ имеет асимптотическое направление), а точка $\sigma$ достигается кривой $\bar{l}$.

Обозначим через $A_{\mathrm{fl}}, A_{\infty}, A_{\mathrm{an}} \subset S_{\infty}$ множества точек, достижимых полутраекториями топологических, $C^{\infty}$ гладких и аналитических потоков на $M$ соответственно. Согласно [2] (см. также [3]), $A_{\mathrm{fl}}=A_{\infty}$.

Геодезическая ламинация есть семейство попарно непересекающихся геодезических, ни одна из которых не имеет трансверсальных самопересечений, и объединение всех геодезических образует замкнутое множество. Любая простая замкнутая геодезическая образует тривиальную геодезическую ламинацию. Обозначим через $\Lambda_{\text {triv }}$ семейство тривиальных геодезических ламинаций на поверхности $M$. Ламинация называется нетривиальной, если она состоит из незамкнутых геодезических. Ламинация минимальна, если она не содержит собственных подламинаций. Нетривиальная минимальная ламинация на $M$ назьвается неприводимой, если любая замкнутая геодезическая на $M$ пересекается с $G$.

Пусть $G$ - геодезическая ламинация на $M$. Введем на геодезических $G$ ориентацию. Введенная ориентация назьвается согласованной, если для любой геодезической $l \in G$ и любой точки $m \in l$ существует отрезок без контакта $\Sigma$ такой, что $m$ принадлежит внутренности $\Sigma$, и все геодезические из $G$, пересекающие отрезок $\Sigma$, пересекают его с одинаковым индексом пересечения. Геодезическая ламинация называется ориентируемой, если на ее слоях можно ввести согласованную ориентацию.

Обозначим через $\Lambda_{\text {or }}$ (соответственно $\Lambda_{\text {non }}$ ) множество нетривиалњных минимальных ориентируемых (соответственно неориентируемых) геодезических ламинаций на $M$. Семейства $\Lambda_{\mathrm{or}}, \Lambda_{\text {non }}$ образуют множество нетривиальных минимальных геодезических ламинаций на $M$,

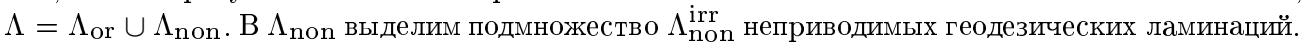

Пусть $G$ - геодезическая ламинация на гиперболической поверхности $M$. Ясно, что прообраз $\pi^{-1}(G) \stackrel{\text { def }}{=} \bar{G}$ является геодезической ламинацией на плоскости $\Delta$. Обозначим через $G(\infty) \subset S_{\infty}$ множество точек абсолюта, достижимых ламинацией $\bar{G}$. Другими словами, $G(\infty)$ есть множество идеальных концевых точек всех геодезических из $\bar{G}$.

Работа выполнена при поддержке Российского фонда фундаментальных исследований (грант № 02-01-00098). 
Основной резултат данной статьи содержится в следующей теореме, которая обобщает результаты работы [8].

ТЕОРема 1. Пусть $M$ - ориентируемая замкнутая гиперболическая поверхность и $S_{\infty}-$ абсолют плоскости Лобачевского $\Delta$, которая является универсальной накрывающей для $M$. Тогда

1) $\Lambda_{\text {triv }}(\infty) \subset A_{\text {an }} \subset \Lambda_{\text {triv }}(\infty) \cup \Lambda_{\text {or }}(\infty)$;

2) $\varnothing \neq \Lambda_{\mathrm{non}}(\infty) \subset A_{\infty}-A_{\mathrm{an}} ;$ множсество $\Lambda_{\mathrm{non}}(\infty)$ континуальное, всюду плотное на $S_{\infty}$ и имеет нулевую меру Лебега на $S_{\infty}$;

3) любой $C^{\infty}$-поток $f^{t}$, достигающий точку из $\Lambda_{\text {non}}(\infty)$, содерэмит бесконечное мнохество точек покоя; более того, если $f^{t}$ достигает точку из $\Lambda_{\mathrm{non}}^{\mathrm{irr}}(\infty)$, то $f^{t}$ содержит континуальное множество точек покоя $u f^{t}$ не имеет ни нетривиально рекуррентных полутраекторий, ни замкнутых негомотопных нулю ииклов без контакта.

Как следствие получаем, что на замкнутой гиперболической ориентируемой поверхности имеется достаточно "болшшое" множество асимптотических направлений, которые реализуются полутраекториями $C^{\infty}$-гладких потоков, но которые не могут быть реализованы полутраекториями никаких аналитических потоков [8].

Для доказателшства основной теоремы доказьвается результат об ограниченности отклонений полутраекторий аналитических потоков, которьй имеет самостоятелшньй интерес. Дадим необходимые определения.

Рассмотрим поднятие $\bar{l}=\{\bar{m}(t): t \geqslant 0\}$ кривой $l$ на $\Delta$. Предположим, что $\bar{l}$ стремится к точке $\sigma$ абсолюта $S_{\infty}$. Пусть $\bar{g}$ - направленная к $\sigma$ геодезическая с идеалшной концевой точкой $\sigma$. Такая геодезическая назьвается представителем асимптотического направления $\sigma$. Кривая $\bar{l}$ обладает свойством ограниченного (соответственно неограниченного) отклонения, если для любой геодезической $\bar{g}$, представляющей асимптотическое направление $\sigma$, отклонение $\bar{l}$ от $\bar{g}$ ограничено (соответственно неограничено).

Ясно, что свойство ограниченного (или неограниченного) отклонения не зависит от выбора представителя асимптотического направления, а для фиксированной кривой $l$ не зависит от выбора поднятия этой кривой. Поэтому можно говорить о свойстве ограниченного (или неограниченного) отклонения кривой $l$ на $M$.

Имеет место следующая теорема.

ТЕОрема 2. Пусть $f^{t}$ - аналитический поток на замкнутой гиперболической ориентируемой поверхности $M$. Тогда любая полутраектория потока $f^{t}$, имеющая асимптотическое направление, обладает свойством ограниченного отклонения.

Для плоских замкнутых поверхностей (тор и бутылка Клейна) аналогичная теорема была доказана Аносовым [3].

Авторы благодарят Д. В. Аносова на полезные обсуждения. Второй автор работал над статьей во время визита в университет г. Рен (Франция), поддержанного CNRS. Он благодарит А. Зорича, И. Итенберга и В. Каймановича за плодотворные дискуссии и тепльй прием.

\section{СПИСОК ЛИТЕРАТУРЫ}

[1] Д. В. Аносов // Изв. АН СССР. Сер. матем. 1987. Т. 51. № 1. С. 16-43. [2] Д. В. Аносов // Изв. АН СССР. Сер. матем. 1988. Т. 52. №3. С. 451-478. [3] Д. В. Аносов // Изв. АН СССР. Сер. матем. 1995. Т. 59. № 2. С. 63-96. [4] С. Х. Арансон, И. А. Гореликова, Е. В. Ж Кжжома // Докл. РАН. 2001. Т. 379. № 2. C. 154-157. [5] S. Aranson, V. Grines, E. Zhuzhoma // Topology. 2001. V. 40. № 3. P. 475-502. [6] D. V. Anosov // J. Dynam. Control Systems. 1995. V. 1. №1. P. 125-138. [7] I. Nikolaev, E. Zhuzhoma. Flows on 2-Dimensional Manifolds. Berlin: Springer-Verlag, 1999. (Lecture Notes in Math. V. 1705.) [8] C. X. Aparсон, Е. В. ЖКужома // Матем. заметки. 2000. Т. 68. №6. С. 819-829. 